В. ОЛЬМАН, А. ШМУНДАК

\title{
МИНИМАКСНОЕ БАИЕСОВСКОЕ ОЦЕНИВАНИЕ СРЕДНЕГО НОРМАЛЬНОГО ЗАКОНА ДЛЯ КЛАССА ОДНОВЕРШИННЫХ АПРИОРНЫХ РАСПРЕДЕЛЕНИЙ
}

\author{
(Представил Н. Алумяэ)
}

Рассмотрим задачу байесовского оценивания случайного параметра $\theta$ нормальной плотности $1 / \sqrt{2 \pi} \exp \left[-(x-\theta)^{2} / 2\right]$ по наблюдению $x$, независимому от $\theta$. Относительно распределения $F$ параметра $\theta$ известно, что $F \in \mathcal{F}(a)\left[{ }^{1}\right]$, т. е. для заданного $a>0$

1) $F(t)+F(-t+0)=1, \quad t>0$,

2) $F(a)=1$,

3) $F(t)$ вогнута при $t>0$.

Подобная постановка рассматривалась в $\left[{ }^{1-4}\right]$, причем в $\left[{ }^{3,4}\right]$ были заданы ограничения на моменты априорного распределения, а в $\left[{ }^{1,2}\right]$, как и в настоящей статье, на его форму.

Определим качество оценки $\delta(x)$ в виде

$$
r(\delta)=\sup _{F \in \mathcal{F}^{(}(a)} R(\delta, F)
$$

где $R(\delta, F)=\int_{-a}^{a} E_{\theta}(\delta(x)-\theta)^{2} d F(\theta)$.

$\mathcal{F}(a)$ - м ин и м а к ной байесовской оценкой назовем $\delta_{0}$ такую, что

$$
\inf _{\delta} r(\delta)=r\left(\delta_{0}\right) \text {. }
$$

В статье исследуется проблема построения $\mathcal{F}(a)$-минимаксной байесовской оценки и дана граница снизу для минимаксного риска $r\left(\delta_{0}\right)$.

Обозначим через $\left\{P_{t}\right\}, a \geqslant t \geqslant 0$, семейство априорных распределений из $\mathcal{F}(a)$, определенных следующим образом:

$$
P_{0}(x)=\left\{\begin{array}{l}
0, x \leqslant 0 \\
1, x>0
\end{array}, \quad P_{t}(x)=\left\{\begin{array}{l}
0, x \leqslant-t \\
\frac{t+x}{2 t},-t<x \leqslant t \\
1 ; x>t
\end{array}\right.\right.
$$

Л ем м 1. Для того, чтобы распределение $F$ принадлежало $\mathcal{F}(a)$, необходимо и достаточно, чтобы существовало такое вероятностное распределение $\mu$, сосредоточенное на $[0, a]$, что

$$
\int_{-a}^{a} u(\theta) d F(\theta)=\int_{0}^{a} \int_{-a}^{a} u(\theta) d P_{t}(\theta) d \mu(t)
$$

для любой непрерывной функциии и $(\theta)$. 
Доказ ательство. Достаточность очевидна в силу линейности интеграла и того, что $P_{t} \in \mathcal{F}(a), 0 \leqslant t \leqslant a$. Для доказательства необходимости заметим, что если $F \in \mathcal{F}(a)$, то существует неотрицательная функция $f(x)$ со свойствами:

1) $f(x)=f(-x), 0 \leqslant x \leqslant a$,

2) $f(x)$ непрерывна справа и не возрастает при $0<x \leqslant a$,

3) $F(x)=\int_{0}^{x} f(t) d t+d F(0), 0<x \leqslant a$.

Определим меру $\mu(t)$ так, что

$d \mu(0)=d F(0), \quad d \mu(t)=-2 t d f(t), \quad 0<t<a, \quad d \mu(a)=2 a f(a-0)$.

Покажем, что определенная так мера является вероятностным распределением, т. е. $\int_{0}^{a} d \mu(t)=1$. Имеем

$$
\int_{0}^{a} d \mu(t)=d F(0)+2 a f(a-0)-\int_{+0}^{a-0} 2 t d f(t)
$$

и, интегрируя по частям, получаем

$$
\int_{0}^{a} d \mu(t)=d F(0)-2\left[a f(a-0)-\lim _{t \rightarrow 0} t f(t)\right]+2 a f(a-0)+2 \int_{+0}^{a-0} d F(t) .
$$

Из непрерывности справа функции $f(t)$ следует существование $\lim _{t \rightarrow 0} t f(t)$. Пусть $\lim _{t \rightarrow 0} t f(t)=c>0$. Тогда для $0<\varepsilon<c$ существует такое $\delta$, что $t f(t)>c-\varepsilon$ при $0<t<\delta$, и следовательно,

$$
\int_{0}^{\delta} f(t) d t>(c-\varepsilon) \int_{0}^{\delta} \frac{1}{t} d t=\infty
$$

что противоречит тому, что $\int_{0}^{\delta} f(t) d t=F(\delta)-F(+0)<\infty$. В силу симметричности распределения $\underset{F}{F}$

$$
2 \int_{+0}^{a-0} d F(t)=\int_{-a+0}^{a-0} d F(t)-d F(0)
$$

что и доказывает утверждение $\int_{0}^{a} d \mu(t)=1$.

Теперь докажем, что для выбранной меры $d \mu(t)$ имеет место равенство (1). Преобразуем правую часть (1) к виду

$$
d F(0) u(0)+\int_{+0}^{a-0} \int_{-t}^{t} u(\theta) d \theta(-d f(t))+f(a-0) \int_{-a}^{a} u(\theta) d \theta
$$

и, интегрируя по частям, получаем

$$
\begin{gathered}
d F(0) u(0)+\left[-\left.f(t) \int_{-t}^{t} u(\theta) d \theta\right|_{+0} ^{a-0}\right]+\int_{+0}^{a-0} f(t)[u(t)+u(-t)] d t+ \\
+f(a-0) \int_{-a}^{a} u(\theta) d \theta .
\end{gathered}
$$

Существование $\lim _{t \rightarrow+0} f(t) \int_{-t}^{t} u(\theta) d \theta$ следует из правосторонней непрерывности функции $f(t)$. Очевидно, 


$$
\left|f(t) \int_{-t}^{t} u(\theta) d \theta\right| \leqslant 2 t f(t) c_{1}(t), \quad c_{1}(t)=\max _{-t \leqslant \theta \leqslant t}|u(\theta)| \leqslant c_{1}(a)
$$

а как доказано ранее, $\lim _{t \rightarrow+0} t f(t)=0$, и следовательно, $\lim _{t \rightarrow+0} f(t) \int_{-t}^{t} u(\theta) d \theta=$ $=0$. Таким образом, правая часть (1) преобразована к виду

$$
d F(0) u(0)+\int_{t 0}^{a-0} f(t)[u(t)+u(-t)] d t,
$$

что совпадает с левой частью равенства (1), а следовательно, лемма доказана.

Введем обозначение $r(\delta, t)=R\left(\delta ; P_{t}\right), 0 \leqslant t \leqslant a$. Тогда в силу леммы 1 существует соответствие между элементами множества $\mathcal{F}(a)$ и пространством $U_{a}$ всех вероятностных мер на $[0, a]$ такое, что

$$
R(\delta, F)=\int_{0}^{a} r(\delta, t) d \mu(t) ;
$$

и таким образом, исходная задача сводится к традиционной минимаксной $\left[{ }^{5}\right]$ с новой функцией риска $r(\delta, t)$

$$
\sup _{0 \leqslant t \leqslant a} r(\delta, t) \longrightarrow \inf _{\delta}
$$

Нетрудно проверить, что для функции риска $r(\delta, t)$ и пространства параметра $t$ допущения $5.1-5.6$, сформулированные А. Вальдом [ $\left.{ }^{5}\right]$, выполнены, а следовательно, имеют место следующие предложения.

Предл ожен и е 1 . Справедливо равенство

$$
\inf _{\delta} \sup _{F \in \mathcal{F}^{(}(a)} R(\delta, F)=\sup _{F \in \mathcal{F}^{(}(a)} \inf _{\delta} R(\delta, F) .
$$

Д ок а з а те ль с т о. В силу выпуклости $r(\delta, t)$ по $\delta$ очевидно равенство

$$
\inf _{\delta} \sup _{\mu \in U_{a}} \int_{0}^{a} r(\delta, t) d \mu(t)=\sup _{\mu \in U_{a}} \inf _{\delta} \int_{0}^{a} r(\delta, t) d \mu(t),
$$

так как $\mu$ является произвольной смешанной стратегией выбора параметра $t$, а используя (2), получаем (3).

О п ределени е. Процедура $\delta$ называется $\mathcal{F}(a)$-допустимой по отношению к $R(\delta, F)$, если не существует процедуры равномерно лучшей, т. е. не существует $\delta_{1}$ такой, что $R(\delta, F) \geqslant R\left(\delta_{1}, F\right) \forall F \in \mathcal{F}(a), \quad$ и хотя бы для одного $F \in \mathscr{F}(a) R(\delta, F)>R\left(\delta_{1}, F\right)$.

Предл ожени е 2. Множество $\mathcal{F}(a)$-допустимых процедур совпадает с классом $B_{a}$ - классом байесовских оценок $\theta$ относительно априорных распределний из $\mathcal{F}(a)$.

Д ок а з т ел ьс т в о. Еслди $\delta-$ байесовская оценка, то она $\mathcal{F}(a)$-допустима, так как для любого распределения $F \in \mathcal{F}($ a) существует единственная байесовская оценка. Пусть теперь $\delta_{1}-\mathcal{F}(a)$-допустимая оценка, но не байесовская по отношению к какому-либо распределению из $\mathcal{F}(a)$, T. e.

$$
\inf _{\delta} R(\delta, F)<R\left(\delta_{1} ; F\right) \quad \forall F \in \mathcal{F}^{*}(a),
$$

что эквивалентно неравенству

$$
\inf _{\delta} \int_{0}^{a} r(\delta, t) d \mu(t)<\int_{0}^{a} r\left(\delta_{1}, t\right) d \mu(t) .
$$


Но в силу теорем А. Вальда $\left[{ }^{5}\right]$ класс байесовских процедур по отношению к $r(\delta, t)$ совпадает с классом допустимых процедур, и следовательно, $\delta_{1}$ не является допустимой по отношению к $r(\delta, t)$. Таким образом, существует оценка $\delta_{2}$ такая, что

$$
r\left(\delta_{2}, t\right) \leqslant r\left(\delta_{1}, t\right), \quad 0 \leqslant t \leqslant a,
$$

а интегрируя последнее неравенство по мерам $\mu \in U_{a}$, получаем противоречие с предположением $\mathcal{F}(a)$-допустимости оценки $\delta_{0}$.

Предложение 3 . $\mathcal{F}(a)$-минимаксная оценка является байесовской по отношению к $F_{0}(x)$ - конечной линейной комбинации распределений $P_{t}(x), 0 \leqslant t \leqslant a$, т. e.

$F_{0}(x)=\sum_{i=1}^{n} \lambda_{i} P_{t_{t}}(x), n \geqslant 1, \sum_{i=1}^{n} \lambda_{i}=1, \lambda_{i} \geqslant 0 ; 1 \leqslant i \leqslant n$.

Доказ а тельство. Очевидно, что для любой байесовской оценки $\delta_{\mu}$ относительно $\mu \in U_{a} r\left(\delta_{\mu}, t\right)-$ аналитическая по $t$ функция. Таким образом, она либо имеет конечное число максимумов на интервале $[0, a]$, либо постоянна. Но если $r\left(\delta_{\mu}, t\right)$ не зависит от $t$, то $R\left(\delta_{\mu}, \theta\right)$ также постоянна по $\theta \in{ }^{\prime}[-a, a]$, а в силу аналитичности и на $(-\infty, \infty)$. Легко показать, что в этом случае $\delta_{\mu}(x)=x$, так как это единственная допустимая на $(-\infty, \infty)$ оценка, имеющая постоянный риск [ $\left.{ }^{6}\right]$. Но $x$ не является допустимой оценкой на $[-a, a]$, а следовательно, $r\left(\delta_{\mu}, t\right)$ имеет лишь конечное число максимумов на интервале [0, $a]$. Как известно $\left[{ }^{5}\right]$, наименее благоприятное по отношению к $r(\delta, t)$ распределение $\mu_{0}$ сосредоточено в точках, соответствующих наибольшему значению функции $r\left(\delta_{\mu_{0}}, t\right)$, и следовательно, существует $n \geqslant 1$ такое, что

$$
F_{0}(x)=\sum_{i=1}^{n} \lambda_{i} P_{t_{i}}(x), \quad \sum_{i=1}^{n} \lambda_{i}=1, \quad \lambda_{i} \geqslant 0,
$$

причем

$$
\sup _{0 \leqslant t \leqslant a} r\left(\delta_{\mu_{0}}, t\right)=r\left(\delta_{\mu_{0}}, t_{i}\right), \quad i=1,2, \ldots, n,
$$

что и доказывает предложение 3.

Л ем м а 2. Для достаточно мальх а байесовская оценка $\delta_{a}$, соответствующсая равномерному на $[-a, a]$ распределению, является $\mathcal{F}(a)$-минимаксной.

Доказательство. Все оценки из класса $B_{a}$ дифференцируемы и суммируемы с квадратом, следовательно,

$$
\frac{d}{d \theta} E_{\theta}(\delta(x)-\theta)^{2}=2 E_{0}\left\{[\delta(x+\theta)-\theta]\left[\delta^{\prime}(x+\theta)-1\right]\right\}, \quad \delta \in B_{a},
$$

причем

$$
2 E_{0}\left[\delta(x+\theta) \delta^{\prime}(x+\theta)\right]=E_{0}\left(\frac{d}{d \theta} \delta^{2}(x+\theta)\right)=E_{0}\left(x \delta^{2}(x+\theta)\right) .
$$

Пусть $\delta_{a}(x)$ - байесовская оценка по отношению к равномерному $[-a, a]$ априорному распределению. Тогда $\delta_{a}(x)$ нечетная функция и при $\theta>0$

$$
E_{0}\left(x \delta^{2}(x+\theta)\right)=\frac{1}{\sqrt{2 \pi}} \int_{0}^{\infty} x\left(\delta^{2}(x+\theta)-\delta^{2}(x-\theta)\right) \exp \left(-x^{2} / 2\right) d x>0,
$$

так как $\delta_{a}(x)$ - возрастающая по $x$ функция. Далее, очевидно, что 


$$
E_{0}\left(\delta(x+\theta)+\theta \delta^{\prime}(x+\theta)\right)=2 \theta E_{0} \delta^{\prime}(x)+O\left(\theta^{3}\right),
$$

и в свою очередь $E_{0} \delta^{\prime}(x)=E_{0} x \delta(x)$. Таким образом, имеем

$$
\frac{d}{d \theta} E_{\theta}\left(\delta_{a}(x)-\theta\right)^{2}=E_{0}\left(x \delta_{a}^{2}(x+\theta)\right)+2 \theta\left[1-E_{0}\left(x \delta_{a}(x)\right)\right]+O\left(\theta^{3}\right),
$$

а так как $\left|\delta_{a}(x)\right| \leqslant a$, то $\left|E_{0} x \delta_{a}(x)\right| \leqslant \sqrt{\frac{2}{\pi}} r$, и следовательно, при достаточно малых $a$ выражение (4) положительно, т. е. $E_{\theta}\left(\delta_{a}(x)-\theta\right)^{2}$ возрастает при $0 \leqslant \theta \leqslant a$. Теперь, используя лемму 3 из $\left[{ }^{2}\right]$, имеем

$$
\sup _{F \in \mathcal{F}^{*}(a)} \int_{-a}^{a} E_{\theta}\left(\delta_{a}(x)-\theta\right)^{2} d F(\theta)=\frac{1}{2 a} \int_{-a}^{a} E_{\theta}\left(\delta_{a}(x)-\theta\right)^{2} d \theta
$$

что с учетом леммы 2 из $\left[{ }^{2}\right]$ завершает доказательство леммы.

3 а м е ч н и е. Численные результаты показали, что монотонность функции $E_{\theta}\left(\delta_{a}(x)-\theta\right)^{2}$ на интервале $[0, a]$ имеет место при $a \leqslant 1 / 2$.

В заключение приведем оценку снизу для минимаксного риска $\inf _{\delta} r(\delta)$.

Т ео рем а. Имеет место неравенство

$$
\inf _{\delta} r(\delta)>r\left(\delta_{a}, a\right) .
$$

Д ока зательст в о. Выпишем последовательность очевидных соотношений:

$$
\begin{gathered}
\inf _{\delta} r(\delta)=\inf _{\delta} \sup _{\mu \in U_{a}} \int_{0}^{a} r(\delta, t) d \mu(t)= \\
=\inf _{\delta} \sup _{0 \leqslant t \leqslant a} r(\delta, t) \geqslant \sup _{0 \leqslant t \leqslant a} \inf _{\delta} r(\delta, t)=\sup _{0 \leqslant t \leqslant a} r\left(\delta_{t}, t\right),
\end{gathered}
$$

где $\delta_{t}-$ байесовская оценка параметра $\theta$ относительно равномерного на $[-t, t]$ распределения. Рассмотрим функцию $r_{1}(s, t)=$ $=r\left(\delta_{s}, t\right)=\frac{1}{2 t} \int_{-t}^{t} R\left(\delta_{s}, \theta\right) d \theta$

Имеем

$$
\frac{d}{d t} r\left(\delta_{t}, t\right)=\left.\frac{\partial r_{1}(s, t)}{\partial s}\right|_{s=t}+\left.\frac{\partial r_{1}(s, t)}{\partial t}\right|_{s=t} .
$$

Но $\left.\frac{\partial r_{1}(s, t)}{\partial s}\right|_{s=t}=0, \quad$ так как $r_{1}(s, t)$ достигает экстремума при $s=t$, т. е. на байесовской оценке. Кроме того,

$$
\left.\frac{\partial r_{1}(s, t)}{\partial t}\right|_{s=t}=\frac{1}{t}\left[R\left(\delta_{t}, t\right)-\frac{1}{2 t} \int_{-t}^{t} R\left(\delta_{t}, \theta\right) d \theta\right]>0
$$

что следует из леммы 6 в $\left[{ }^{1}\right]$, таким образом, $\frac{d}{d t} r\left(\delta_{t}, t\right)>0$; а следовательно,

$$
\sup _{0<t \leqslant a} r\left(\delta_{t}, t\right)=r\left(\delta_{a}, a\right),
$$

что и доказывает теорему. 


\section{ЛИТЕРАТУ РӒ}

1. Шмундак А., Ольман В. Йзв. АН ЭССР. Физ. Матем., 34, № 1, 20-25 (1985).

2. Ольман В. Изв. АН ЭССР. Физ. Матем., 33, № 3, 285-290 (1984).

3. Skibinsky, M. Ann. Math. Statist., 39, № 2, 492-501 (1968).

4. Skibinsky, M. SIAM J. Appl. Math., 16, 134-145 (1968).

5. Вальд А. Статистические решающие функции. В кн.: Позиционные игры. М., «Наука», 1967.

6. Каган А. М., Линник Ю. В., Рао С. F. Характеризационные задачи математической статистики. М., «Наука», 1972.

Институт кибернетики

Академии наук Эстонской ССР
Поступила в редакцию

12/XII 1983

\section{OLMAN, A. SMUNDAK}

\section{NORMAALJAOTUSE KESKVĂARTUSE BAYESI MINIMAKSHINNANG UNIMODAALSETE APRIOORSETE JAOTUSTE KLASSILE}

On vaadeldud normaaljaotuse nihkeparameetri $\theta$ minimakshinnangut tingimusel, ef parameetri aprioorne jaotus kuulub sümmeetriliste unimodaalsete ja lôplikul intervallil $[-a, a]$ jaotatud jaotuste klassi $\mathcal{F}(a)$, s.t. on vaadeldud $\underset{F \in \mathcal{F}(a)}{\sup } r(\delta, F)$ minimeerimist, kus $r(\delta, F)=\int_{-a}^{a} E_{\theta}(\delta(x)-\theta)^{2} d F(\theta), \quad x \sim N(\theta, 1)$. On näidatud, et väikeste $a$ väärtuste korral lahendab ülesande Bayesi hinnang $\delta_{a}$, mis vastab aprioorsele ühtlasele jaotusele vahemikus $[-a, a]$, ning tōestatud, et riskifunktsiooni $r(\delta, F)$ suhtes lubatavate protseduuride klass ühtib Bayesi hinnangute alamklassiga, mis vastab $\mathscr{F}(a)$ elementidele. Suvalise $a>0$ jaoks on leitud alumine minimakshinnang.

\section{OLMAN, A. SHMUNDAK}

\section{MINIMAX BAYES ESTIMATION OF MEAN OF NORMAL LAW FOR THE CLASS OF UNIMODAL A PRIORI DISTRIBUTIONS}

The problem of Bayes estimation of the mean value $\theta$ of normal distribution is considered. It is assumed that the a priori distribution $F(\theta) \in \mathcal{F}(a)$, i.e. $F(\theta)$ is 1) symmetrical, 2) concentrated on the interval $[-a, a]$ where $a$ is a fixed positive, $3)$ convex on $[-a, 0]$. The problem is to minimize sup $r(\delta, F)$, where $r(\delta, F)=$ $=\int_{-a}^{a} E_{\theta}(\delta(x)-\theta)^{2} d F(\theta), \quad x \sim N(\theta, 1)$. It is shown that for any sufficiently small $a>0$ the Bayes estimator $\delta_{a}$ corresponding to the uniform distribution on $[-a, a]$ provides a solution of this problem. It is proved that the class of procedures admissible with respect to the risk function $r(\delta, F)$, coincides with the set of all Bayes estimators corresponding to distributions from $\mathscr{F}(a)$. The lower bound for minimax risk for any $a>0$ is given. 